OPEN ACCESS

Edited by:

Djoher Nora Abrous,

Institut des Neurosciences de

Bordeaux, France

Reviewed by:

Serge H. Ahmed,

Centre National de la Recherche

Scientifique, France

Stéphanie Caille,

Centre National de la Recherche

Scientifique, France

*Correspondence:

Selena E. Bartlett

selena.bartlett@qut.edu.au

Received: 03 December 2015

Accepted: 07 March 2016

Published: 23 March 2016

Citation:

Klenowski PM, Shariff MR, Belmer A,

Fogarty MJ, Mu EWH, Bellingham MC and Bartlett SE (2016) Prolonged

Consumption of Sucrose in a

Binge-Like Manner, Alters the

Morphology of Medium Spiny

Neurons in the Nucleus Accumbens

Shell. Front. Behav. Neurosci. 10:54.

doi: 10.3389/fnbeh.2016.00054

\section{Prolonged Consumption of Sucrose in a Binge-Like Manner, Alters the Morphology of Medium Spiny Neurons in the Nucleus Accumbens Shell}

\author{
Paul M. Klenowski ${ }^{1}$, Masroor R. Shariff ${ }^{1}$, Arnauld Belmer ${ }^{1}$, Matthew J. Fogarty ${ }^{2}$, \\ Erica W. H. Mu ${ }^{2}$, Mark C. Bellingham ${ }^{2}$ and Selena E. Bartlett ${ }^{1 *}$ \\ ${ }^{1}$ Translational Research Institute and Institute for Health and Biomedical Innovation, Queensland University of Technology, \\ Brisbane, QLD, Australia, ${ }^{2}$ School of Biomedical Sciences, The University of Queensland, Brisbane, QLD, Australia
}

The modern diet has become highly sweetened, resulting in unprecedented levels of sugar consumption, particularly among adolescents. While chronic long-term sugar intake is known to contribute to the development of metabolic disorders including obesity and type II diabetes, little is known regarding the direct consequences of long-term, binge-like sugar consumption on the brain. Because sugar can cause the release of dopamine in the nucleus accumbens (NAc) similarly to drugs of abuse, we investigated changes in the morphology of neurons in this brain region following short- (4 weeks) and long-term (12 weeks) binge-like sucrose consumption using an intermittent two-bottle choice paradigm. We used Golgi-Cox staining to impregnate medium spiny neurons (MSNs) from the NAc core and shell of short- and long-term sucrose consuming rats and compared these to age-matched water controls. We show that prolonged binge-like sucrose consumption significantly decreased the total dendritic length of NAc shell MSNs compared to age-matched control rats. We also found that the restructuring of these neurons resulted primarily from reduced distal dendritic complexity. Conversely, we observed increased spine densities at the distal branch orders of NAc shell MSNs from long-term sucrose consuming rats. Combined, these results highlight the neuronal effects of prolonged binge-like intake of sucrose on NAc shell MSN morphology.

Keywords: binge-like consumption, long-term, medium spiny neuron, nucleus accumbens, sucrose

\section{INTRODUCTION}

Over the last 40 years, there has been a documented rise in the consumption of sugar-sweetened beverages and foods containing added sugars (Nielsen et al., 2002; Popkin, 2010; Ng et al., 2012), with reports estimating that up to $75 \%$ of all foods and beverages contain high amounts of added sugars (Ford and Dietz, 2013; Bray and Popkin, 2014). During this period, there has also been a concurrent increase in the prevalence of obesity and type II diabetes, particularly among adolescents (Arslanian, 2002; Reinehr, 2013; Dabelea et al., 2014; Fryar et al., 2014). Recent studies have shown that overweight and obese children often consume high amounts of added sugar, however the contribution of high sugar containing diets to the increased incidence of overweight 
and obese children remains controversial ( $\mathrm{Hu}, 2013$; Bray and Popkin, 2014; Bucher Della Torre et al., 2015).

While a growing body of evidence indicates that the consumption of high sugar diets may, in part, contribute to weight gain among children and adolescents (Malik et al., 2010; Te Morenga et al., 2013; Bray and Popkin, 2014), less attention has been given to adverse non-metabolic consequences arising from excessive sugar intake. Interestingly, some common behavioral and psychological patterns often emerge amongst a subset of those who over eat and maintain high sugar containing diets. Most notable are the development of eating disorders including binge-eating, combined with the concurrent onset of psychological symptoms including lack of motivation and depression (reviewed in Sheehan and Herman, 2015). In addition, because binge-eating individuals often exhibit a loss of control and an inability to self-limit their sugar intake, it is likely that these behaviors arise as a result of neurological adaptations in brain regions that evaluate the hedonic value of highly palatable food (Saper et al., 2002; Lutter and Nestler, 2009; Kenny, 2011). This rationale is also supported by evidence in humans demonstrating that sugar and sweetness can cause cravings that are similar to those induced by addictive drugs such as alcohol and nicotine (Volkow et al., 2012).

Although the addictive properties of sugar are still speculative, these observations combined with studies demonstrating the contribution of excessive sugar intake to changes in reward circuitry and the development of addictive-like behaviors and emotional states in animal models (Avena et al., 2008; Benton, 2010; Ventura et al., 2014), warrants the need for further investigation. Previous studies in rodents have shown that intermittent access to sucrose alters the activity of several neurotransmitters within the mesolimbic system including dopamine, opioids and acetylcholine (Reviewed in Avena et al., 2008). Binge-like consumption of sucrose has been shown to facilitate dopamine release in the nucleus accumbens (NAc), similarly to drugs of abuse (Avena et al., 2008). Furthermore, we have shown that long-term consumption of sucrose using a $24 \mathrm{~h}$ intermittent access two-bottle choice paradigm (Simms et al., 2008) modulates nicotinic acetylcholine receptor (nAChR) expression in the NAc (Shariff et al., in press). Interestingly, we have also observed that $\mathrm{nAChR}$ compounds known to modulate dopamine and acetylcholine activity in the NAc, have different effects on sucrose consumption following short- and long-term intake (Shariff et al., in press).

While these studies have demonstrated similarities in the behavioral and neurochemical changes caused by intermittent access to sugar and drugs of abuse, it is not known whether these effects facilitate changes in neuronal morphology in the NAc. This is in contrast to substances of abuse including cocaine, amphetamine and nicotine which produce well characterized changes in the morphology of medium spiny neurons (MSNs) in the NAc, including increased spine density and altered dendritic complexity (Robinson and Kolb, 1999, 2004; Li et al., 2003; Crombag et al., 2005). Because we have previously shown that long-term exposure (12 week) to alcohol and sucrose using the intermittent two-bottle choice paradigm produces a differential response to pharmacotherapeutic interventions compared to short-term intake (4 weeks; Steensland et al., 2007; Shariff et al., in press), we assessed the effects of short- and long-term sucrose consumption on MSN morphology in the NAc. We allowed adolescent rats to consume sucrose in a binge-like manner for 4 (short-term) or 12 (long-term) weeks and then analyzed the morphology of NAc MSNs from short- and long-term sucrose consuming rats and compared this to age-matched controls who were given access to water only. Our results show that MSNs from the NAc shell are altered following long- but not shortterm sucrose consumption, having reduced dendritic length, but increased distal dendritic spine density. Furthermore, we found the morphology of MSNs from the NAc core remained relatively intact following short- and long-term sucrose consumption. These results highlight a direct neurological consequence of longterm sucrose consumption in a binge-like manner. Furthermore, this data demonstrates the need for further studies aimed at elucidating the molecular and neurochemical changes that accompany the morphological restructuring of NAc shell MSNs induced by prolonged, binge-like sucrose intake.

\section{MATERIALS AND METHODS}

\section{Ethics Statement}

All experimental procedures were carried out in accordance with the Australian Code for the Care and Use of Animals for Scientific Purposes, 8th Edition (National Health and Medical Research Council, 2013). The protocols were approved by the Queensland University of Technology Animal Ethics Committee and the University of Queensland Animal Ethics Committee.

\section{Animals and Housing}

Five-week-old (adolescent) male Wistar rats (Control: $176.4 \pm$ $4.8 \mathrm{~g}$; Sucrose: $178.3 \pm 5.0 \mathrm{~g}$ ) (ARC, WA, Australia), were individually housed in ventilated dual level Plexiglas ${ }^{\circledR}$ cages. The rats were acclimatized to the individual housing conditions, handling, and reverse-light cycle 5 days before the start of the experiments. All rats were housed in a climate-controlled 12-hr reversed light/dark cycle (lights off at 9 a.m.) room with standard rat chow and water available ad libitum.

\section{Intermittent-Access Two-Bottle Choice Drinking Paradigm}

The intermittent access 5\% sucrose two-bottle choice drinking paradigm (Simms et al., 2008) was adapted from Wise (1973). All fluids were presented in $300 \mathrm{ml}$ graduated plastic bottles with stainless-steel drinking spouts inserted through two grommets in the front of the cage following the commencement of the dark light cycle. Weights of each bottle were recorded prior to bottle presentation. Two bottles were presented simultaneously: one bottle containing water; the second bottle containing $5 \%$ $(\mathrm{w} / \mathrm{v})$ sucrose. The placement of the $5 \%(\mathrm{w} / \mathrm{v})$ sucrose bottle was alternated with each exposure to control for side preferences. Bottles were weighed $24 \mathrm{~h}$ after the fluids were presented, and measurements were taken to the nearest $0.1 \mathrm{~g}$. The weight of each rat was also measured to calculate the grams of sucrose intake per kilogram of body weight. On day 1 of the drinking period, rats $(n=6-9)$ were given access to one bottle of $5 \%(\mathrm{w} / \mathrm{v})$ sucrose and one bottle of water. After $24 \mathrm{~h}$, the sucrose bottle was replaced with a second water bottle that 
was available for the next $24 \mathrm{~h}$. This pattern was repeated on Wednesdays and Fridays. The rats had unlimited access to water on all other days. Binge-like consumption of sucrose resulted in an escalation in total sucrose intake (ml) over time (Supplementary Figure 1) and was accompanied by stable baseline drinking levels based on body weight $[20 \pm 5 \mathrm{~g} / \mathrm{kg}$ of the $5 \%(\mathrm{w} / \mathrm{v})]$ during the short-term $[\sim 4$ weeks (13 drinking sessions)] and long-term [ $\sim 12$ weeks (37 drinking sessions)] drinking periods. A separate group of control rats $(n=6-9)$ were given access to water in both bottles (i.e., no sucrose) under the same conditions described above. The mean body weight of control and sucrose consuming rats at the end of short-term exposure was $405.7 \pm 40.8 \mathrm{~g}$ and $426.4 \pm 31.2 \mathrm{~g}$ respectively. At the end of long-term exposure, the mean body weight for control and sucrose groups was $578.8 \pm 53.4 \mathrm{~g}$ and $600.2 \pm$ $45.2 \mathrm{~g}$.

\section{Golgi-Cox Staining}

Following the last drinking session rats were transferred from the animal facility to allow for processing of the brain samples at the histology facility at the School of Biomedical Sciences, University of Queensland (St Lucia, Australia). All approved measures were taken to reduce stress during transport, following which, rats were allowed to recover overnight. The next day, rats were sacrificed by sodium pentobarbital overdose $(60-80 \mathrm{mg} / \mathrm{kg}$, i.p. Vetcare, Brisbane, Australia) and intracardially perfused with $\sim 300 \mathrm{ml}$ artificial cerebro-spinal fluid that contained, (in $\mathrm{mM}$ ): $130 \mathrm{NaCl}, 3 \mathrm{KCl}, 26 \mathrm{NaHCO}_{3}, 1.25 \mathrm{NaH}_{2} \mathrm{PO}_{4}, 5 \mathrm{MgCl}_{2}$, $1 \mathrm{CaCl}_{2}$, and $10 \mathrm{D}$-glucose. Each animal was then decapitated and the brain removed and incubated in the dark in Golgi-Cox solution that contained $5 \%$ potassium dichromate, $5 \%$ potassium chromate, and 5\% mercuric chloride (all chemicals from SigmaAldrich) that was made fresh 3 days prior to sacrifice as described previously (Rutledge et al., 1969). Golgi-Cox stain incubation and post-processing methods were modified from Ranjan and Mallick (2010). Brains from short-term sucrose consuming animals were incubated for 6 days at $37^{\circ} \mathrm{C}$, whilst brains from long-term sucrose consuming animals were incubated for 10 days, with one change to fresh Golgi-Cox solution after 4 days of incubation.

Following incubation, $300 \mu \mathrm{m}$ coronal sections were cut using a vibrating Zeiss Hyrax V50 microtome (Carl Zeiss, Germany). Slices were then placed sequentially in 24-well plates filled with $30 \%(\mathrm{w} / \mathrm{v})$ sucrose in $0.1 \mathrm{M}$ phosphate buffered saline and processed as outlined in (Ranjan and Mallick, 2010). Briefly, sections were dehydrated in $50 \%$ ethanol for $5 \mathrm{~min}$, then placed in $0.1 \mathrm{M} \mathrm{NH}_{4} \mathrm{OH}$ solution for $30 \mathrm{~min}$, rinsed twice with distilled water for $5 \mathrm{~min}$ and placed in Fujihunt film fixer (Fujifilm, Singapore) for $30 \mathrm{~min}$ in the dark. The slices were then rinsed twice in distilled water for 2 min each and dehydrated in 70, 90, 95, and $100 \%$ ethanol twice for $5 \mathrm{~min}$ each. The sections were then cleared in CXA solution (1:1:1 chloroform:xylene:alcohol) for $10 \mathrm{~min}$ and mounted in DPX (Sigma-Aldrich) on Superfrost Plus slides (Menzel-Glaser, Lomb Scientific, Australia) and cover-slipped (Menzel-Glaser, Germany). The slides were left in the dark to dry at room temperature overnight.

\section{Neuronal Selection and Tracing within the Nucleus Accumbens}

Coronal slices between bregma +2.8 and +1.7 were surveyed for MSNs within the core and shell of the NAc, using the lateral ventricle and the anterior commissure as landmarks with the aid of a rat brain atlas (Paxinos and Watson, 2007) (Figure 1). The contour function in Neurolucida 7 (MBF Bioscience, VT, USA) was used to demarcate the NAc core and NAc shell in each slice (Figure 2). Between 2 and 9 neurons per region per animal were traced for dendritic length parameters using a $63 \mathrm{x}$ objective or for spine densities (reported as spines per $100 \mu \mathrm{m}$ ) using a 100x objective on a Zeiss Axioskop II (Carl Zeiss, Germany) using an automated $x y z$ stage driven by Neurolucida ${ }^{\circledR} 7$ software (MBF Biosciences, VT, USA). All tracing was performed in a blinded fashion with respect to treatment. Morphological parameters of Golgi-Cox impregnated neurons were analyzed in a manner similar to previous reports (Klenowski et al., 2015).

\section{Statistical Analysis}

Mean and standard error of the mean (SEM) were calculated for each data set with the animal as $n$, using the mean morphometry

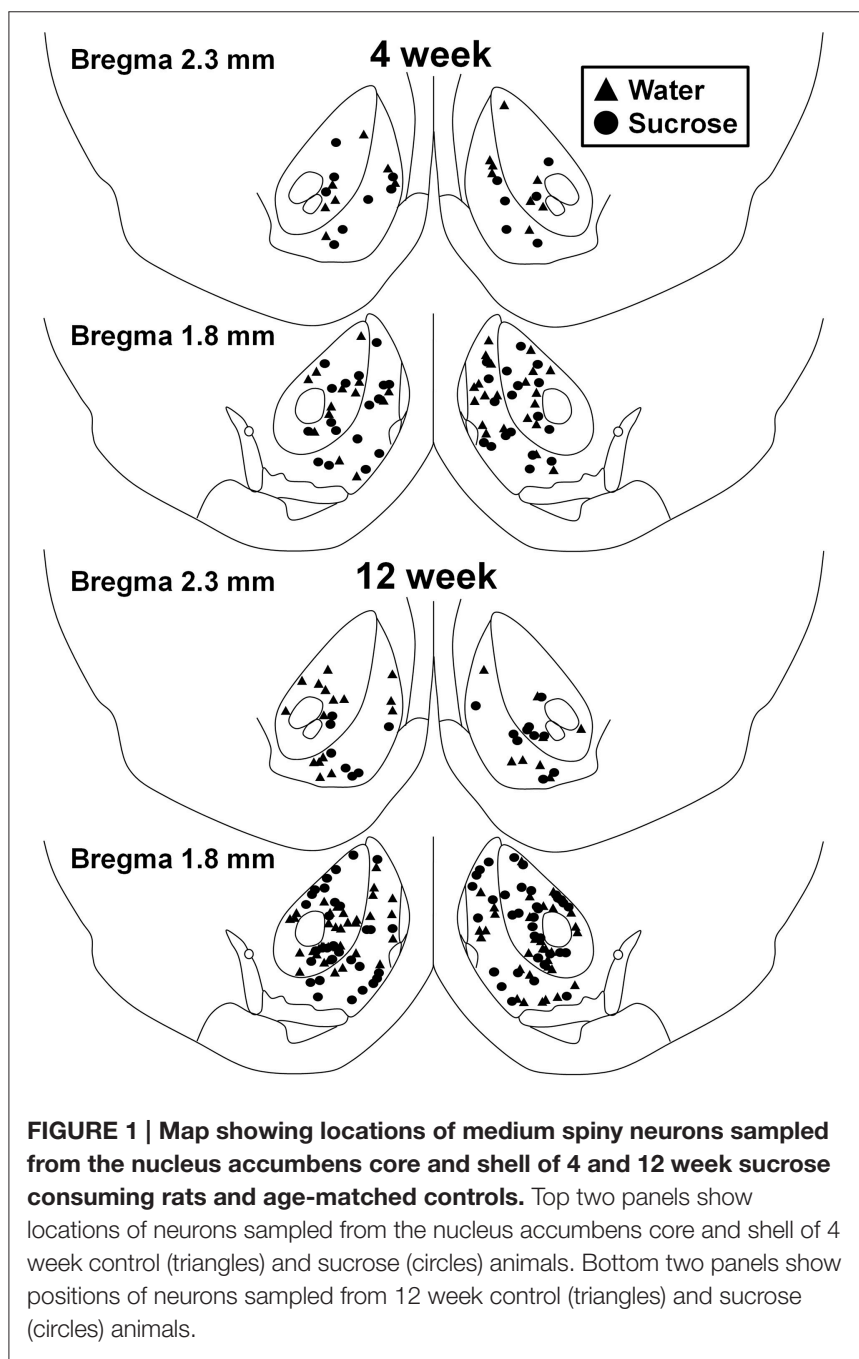


data from all the core or shell NAc MSNs $(n=7$ for NAc shell and $n=6$ for NAc core 4 -week, $n=9$ for 12-week groups). Where indicated, unpaired two tailed Student's $t$-tests or two-way ANOVAs with Bonferroni post-tests were conducted for all analyses involving the comparison of group means, using GraphPad Prism version 6.02 (GraphPad Software, San Diego, CA). Statistical significance was accepted at $P<0.05$. All data in the results section are presented as means \pm SEM. Percentage changes are calculated as relative to the control value.

\section{RESULTS}

\section{Medium Spiny Neurons from the Nucleus Accumbens Shell have Decreased Dendritic Length, Decreased Dendritic Complexity but Increased Mean Spine Density at Distal Branch Orders Following Long- but not Short-Term Sucrose Consumption}

Following short-term (4 weeks) sucrose consumption, there were no significant differences in NAc shell MSN morphometric parameters (Table 1). There were also no significant differences between short-term sucrose consumption and water control NAc shell MSNs in analyses related to centrifugal branch order. Namely, dendritic segments per branch order $(P=0.4111)$, mean dendritic length per branch order $(P=0.5581)$ and mean spine density per branch order $(P=0.2977$, two-way ANOVAs $)$ were not significantly different between groups. A location map showing the approximate positions of the sampled neurons is shown in Figure 1.

Following long-term (12 weeks) of sucrose consumption, the total dendritic arbor length of NAc shell MSNs were decreased by $21 \%$ compared to water consuming controls (Water: $1827 \pm 148$ $\mu \mathrm{m}, n=9$; Sucrose $1449 \pm 78 \mu \mathrm{m}, n=9,{ }^{*} P=0.0384$, two-tailed unpaired Student's $t$-test, Figure 2, Table 2). Comparison of the mean number of dendritic bifurcations (nodes) and dendritic endings between the water and sucrose groups revealed a reduced (although not significant) level of dendritic complexity in NAc shell MSNs (nodes: Water $12.9 \pm 1.4 n=9$, Sucrose $10.1 \pm$ $0.8 n=9, P=0.0879$; endings: Water $17.9 \pm 1.4 n=9$,

TABLE 1 | General morphologic parameters of medium spiny neurons from the nucleus accumbens shell of short-term sucrose consuming rats and age-matched water controls.

\begin{tabular}{lccc}
\hline Parameter & Water $(\boldsymbol{n})$ & Sucrose $(\boldsymbol{n})$ & $\boldsymbol{P}$-value \\
\hline Soma Volume $\left(\mu \mathrm{m}^{3}\right)$ & $2337 \pm 149(7)$ & $2216 \pm 132(7)$ & 0.5606 \\
Total Dendrite Length $(\mu \mathrm{m})$ & $1640 \pm 159(7)$ & $1515 \pm 189(7)$ & 0.6217 \\
Mean Tree Length $(\mu \mathrm{m})$ & $422 \pm 22(7)$ & $392 \pm 32(7)$ & 0.4510 \\
Nodes & $10.9 \pm 1.1(7)$ & $9.9 \pm 1.1(7)$ & 0.4223 \\
Endings & $14.3 \pm 1.4(7)$ & $14.7 \pm 0.7(7)$ & 0.8136 \\
Spines Per $100 \mu \mathrm{m}$ & $54.5 \pm 5.0(7)$ & $57.1 \pm 2.5(7)$ & 0.6356 \\
\hline
\end{tabular}

All data presented as mean \pm SEM. Two-tailed unpaired Student's t-tests.
Sucrose $14.8 \pm 0.7 n=9, P=0.0657$, two-tailed unpaired Student's $t$-test, Table 2$)$. There was no change in soma volume $(P=0.9400)$, mean dendritic tree length $(P=0.1646)$ or total spine density $(P=0.3662)$ in NAc shell MSNs from longterm sucrose consuming rats compared to water controls. These morphometric parameters are detailed in Table 2 .

Following the characterization of the general dendritic morphology of long-term sucrose consuming NAc shell MSNs, we analyzed dendritic arborizations and spine densities with regard to their branch order characteristics. Our comprehensive assessment of the dendritic trees quantified the number of dendritic segments per branch order, the mean length of dendritic segments per branch order and mean spine density per branch order of NAc shell MSNs of water control and long-term sucrose consuming rats. A summary of the branch order data and analysis is presented in Table 3.

The mean dendritic branch segment number per branch order of NAc shell MSNs was significantly reduced in long-term sucrose consuming rats compared to water controls $\left({ }^{* *} P=0.0015\right.$, twoway ANOVA). Bonferroni post-tests revealed a trend toward a reduced number of branch segments at 4 th (Water: $5.2 \pm 0.9$, $n=9$; Sucrose $3.3 \pm 0.8, n=9, P=0.0675$, Figure 2F, Table 3), and 5th order and above branch orders (Water: $3.3 \pm 0.7, n=$ 9; Sucrose 1.2 $\pm 0.3, n=9, P=0.0566$, Figure 2F, Table 3). The mean dendritic segment length per branch order of NAc shell MSNs was also significantly reduced in long-term sucrose consuming rats compared to water controls $\left({ }^{*} P=0.0444\right.$, twoway ANOVA). Bonferroni post-tests showed a reduction of 55\% at 5th order branches and beyond (Water: $53.9 \pm 7.2 \mu \mathrm{m}, n$ = 9; Sucrose $24.1 \pm 7.5 \mu \mathrm{m}, n=9,{ }^{* *} P=0.0038$, Figure 2G, Table 3).

Branch order analysis showed a significant increase in the dendritic spine density of NAc shell MSNs of long-term sucrose consuming rats compared to controls $\left({ }^{*} P=0.0124\right.$, twoway ANOVA). Bonferroni post-tests showed a spine density increase of $57 \%$ at distal 4 th order branches and beyond (Water: $33.4 \pm 4.2, n=9$; Sucrose $52.5 \pm 6.8, n=9, P=0.0271^{*}$, inset of Figures 2A,B,H, Table 3). Representative images of overall MSN architecture and distal spine density (inset) are depicted in Figures 2A,B.

Taken together, these results indicate that short-term sucrose consumption has little effect on morphologic parameters of MSNs within the NAc shell. However, following prolonged consumption, there is a significant decrease in the neuronal arbor length and complexity, particularly in distal dendritic branches. Concomitant distal spine density increases are also apparent in NAc shell MSNs of long-term sucrose consuming rats.

\section{Medium Spiny Neurons from the Nucleus Accumbens Core have Reduced Branching Complexity after Long- but not Short-Term Sucrose Consumption}

Following short-term of sucrose consumption, there were no significant differences in NAc core MSN morphometric parameters (Table 4). There were also no significant differences between 4-week sucrose consumption and water control core 

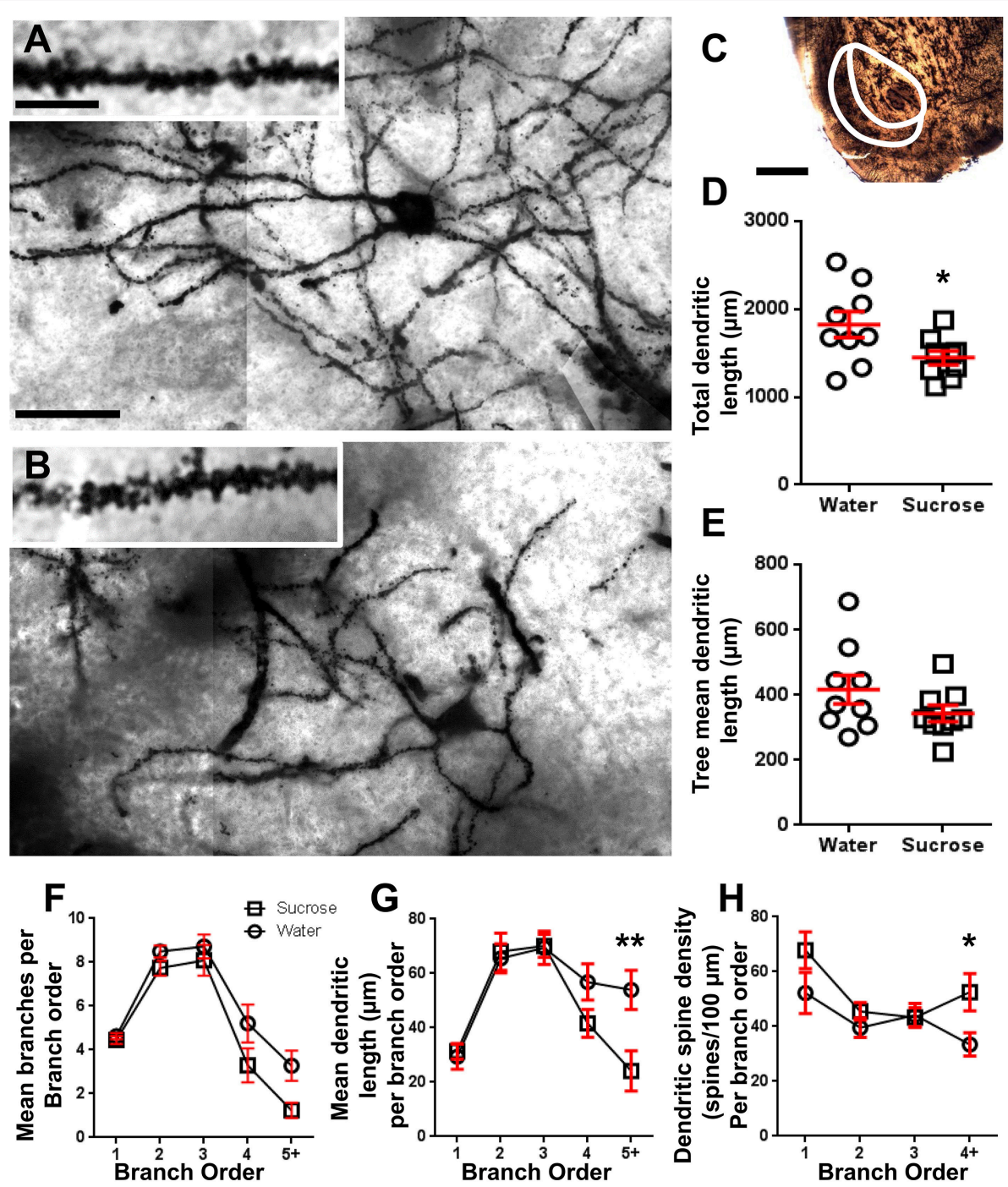

FIGURE 2 | Decreased dendritic arbor length and increased distal dendritic spine density of medium spiny neurons (MSNs) from the nucleus accumbens (NAc) shell of long-term sucrose treated rats compared to control rats. (A,B) show representations of control (top) and long-term (12 week) sucrose (bottom) treated brightfield z-stack mosaics of Golgi-impregnated MSNs from the NAc shell (63x magnification). Inset of (A,B) shows control and long-term sucrose treated brightfield images of Golgi-impregnated MSN dendrites and dendritic spines from the NAc shell (100x magnification). (C) shows the anatomical regions that MSNs were sampled from in this study. (D) shows a scatter-plot of decreased total MSN dendritic arbor (mean \pm SEM) from the NAc shell in long-term sucrose animals (squares) compared to controls (circles), unpaired students $t$-test, ${ }^{\star} P<0.05, n=9$; control and $n=9$; 12 week sucrose. (E) shows a scatter-plot of unchanged mean MSN dendritic tree length (mean \pm SEM) from the NAc shell in long-term sucrose animals (squares) compared to controls (circles), unpaired students $t$-test, $P>0.05, n=9$; control and $n=9 ; 12$ week sucrose. Branch order analysis (mean \pm SEM) of dendritic segment number per branch order (F), mean dendritic length per branch order $\mathbf{( G )}$ and dendritic spine density per branch order $\mathbf{( H )}$. Long-term sucrose consumption decreased dendritic length at distal branch orders (5+) and increased dendritic spine density at distal branch orders (4+) compared to controls $(\mathbf{G}, \mathbf{H})$, two-way ANOVAs with Bonferroni post-tests, ${ }^{\star} P<0.05$, ${ }^{\text {** }} P<0.01, n=9$; control and $n=9$; long-term sucrose. Scale Bars: $(\mathbf{A}, \mathbf{B})=20 \mu \mathrm{m}$; inset of $(\mathbf{A}, \mathbf{B})=10 \mu \mathrm{m} ;(\mathbf{C})=1 \mathrm{~mm}$.

MSNs in analyses related to centrifugal branch order. Namely, dendritic segments per branch order $(P=0.7717)$, mean dendritic length per branch order $(P=0.2096)$, and mean spine density per branch order $(P=0.3521$, two-way ANOVAs) were not different between groups.

Prolonged sucrose consumption also had no significant on NAc core MSN morphometric parameters (Table 5). The mean dendritic branch segment number per branch order of NAc core MSNs was significantly reduced in long-term sucrose consuming rats compared to water controls $\left({ }^{*} P=0.0416\right.$, twoway ANOVA), however there were no significant differences in mean dendritic length per branch order $(P=0.0995)$ and mean spine density per branch order $(P=0.4888$, two-way ANOVAs) between MSNs in the NAc core of long-term sucrose consuming rats compared to water controls. Taken together, our data shows the NAc core is not as responsive to long-term sucrose consumption compared to MSNs from the NAc shell region. 
TABLE 2 | General morphologic parameters of medium spiny neurons from the nucleus accumbens shell of long-term sucrose consuming rats and age-matched water controls.

\begin{tabular}{lccr}
\hline Parameter & Water $(\boldsymbol{n})$ & Sucrose $(\boldsymbol{n})$ & $\boldsymbol{P}$-value \\
\hline Soma Volume $\left(\mu \mathrm{m}^{3}\right)$ & $4508 \pm 387(9)$ & $4453 \pm 599(9)$ & 0.9400 \\
Total Dendrite Length $(\mu \mathrm{m})$ & $1827 \pm 148(9)$ & $1449 \pm 78(9)$ & ${ }^{*} 0.0384$ \\
Mean Tree Length $(\mu \mathrm{m})$ & $417 \pm 44(9)$ & $343 \pm 25(9)$ & 0.1646 \\
Nodes & $12.9 \pm 1.4(9)$ & $10.1 \pm 0.8(9)$ & 0.0879 \\
Endings & $17.9 \pm 1.4(9)$ & $14.8 \pm 0.7(9)$ & 0.0657 \\
Spines Per 100 $\mu \mathrm{m}$ & $40.4 \pm 3.3(9)$ & $45.0 \pm 3.7(9)$ & 0.3662 \\
\hline
\end{tabular}

All data presented as mean \pm SEM. ${ }^{*} P<0.05$, two-tailed unpaired Student's $t$-tests.

\section{DISCUSSION}

The increased availability of highly sweetened food in the Western diet has not only contributed to the increased prevalence and economic burden of obesity and type II diabetes, it has also led to an onset of eating disorders such as binge eating (Swanson et al., 2011; Kessler et al., 2013; Davis, 2015). Although the addictive properties of sugars including fructose and sucrose remain speculative, there is a striking similarity in the behavioral and neural correlates that manifest as a result of over eating and prolonged drug use (Avena et al., 2008, 2011). In addition, sugar activates the brain's reward circuitry in a way that is similar to drugs of abuse (Volkow et al., 2012), and results from human studies suggest that sugar and sweetness can induce cravings that are comparable in magnitude to those induced by addictive drugs such as alcohol and nicotine (Volkow et al., 2012). We therefore used a model of binge-sucrose consumption in rats to determine the effects of short- (4 weeks) and long-term (12 weeks) sucrose consumption on neuronal morphology of MSNs in the NAc, a key component of the overlapping reward circuitry that is modulated by sugar and addictive drugs. We show that MSNs from the NAc shell of chronic long-term sucrose consuming rats have significantly decreased dendritic length and complexity, but increased distal dendritic spine density. Long-term sucrose consumption had no effect on the morphology of MSNs from the NAc core, while short-term sucrose consumption also had no significant effect on MSN morphology from the NAc core or shell. These results not only demonstrate a direct effect of prolonged binge-like sucrose intake on neuronal morphology of NAc shell MSNs, but they also highlight the potentially harmful consequences of the prolonged consumption of high sugar containing diets.

The NAc, which forms part of the ventral striatum, is comprised primarily of MSNs, which are morphologically characterized as medium-sized neurons with extensive dendritic arborisations and high spine density (Kemp and Powell, 1971; Graveland and DiFiglia, 1985; Rafols et al., 1989; Kawaguchi et al., 1990). Glutamatergic and dopaminergic neurons are the two primary afferent inputs to the NAc, primarily contacting the dendritic shafts and spines of MSNs (Groves, 1980; Kaiya and Namba, 1981; Groves et al., 1994). Specifically, the NAc shell and core receive glutamatergic input from functionally distinct cortical areas (Brog et al., 1993). The NAc shell is also innervated by excitatory afferents from subcortical regions such as the hippocampus, thalamus and basolateral amygdala (Brog et al., 1993; Wright and Groenewegen, 1995). Previous studies have demonstrated that these glutamatergic inputs play a pivotal role in motivation and goal-directed behaviors such as food and reward seeking (Maldonado-Irizarry et al., 1995; Kelley and Swanson, 1997; Reynolds and Berridge, 2003; Richard and Berridge, 2011). The other predominant input onto NAc MSNs is from dopaminergic afferents that project from the ventral tegmental area (Lindvall and Björklund, 1978; Veening et al., 1980; Kalivas and Miller, 1984). Interestingly, previous studies using similar models of intermittent sugar access have shown that the resulting binge-like consumption results in an increase in extracellular dopamine in the NAc similarly (albeit to a lesser extent) to drugs of abuse (Rada et al., 2005; Avena et al., 2006), and can modulate dopamine receptor expression (Colantuoni et al., 2001, 2002) in the NAc core and shell. Interestingly, bingelike consumption of sucrose causes an escalation in intake over time similarly to self-administration of drugs of abuse such as cocaine and heroin (Ahmed and Koob, 1998; Ahmed et al., 2000, 2003) which is associated with the development of an "addictive like" state.

Our analysis of branch order morphometry shows that the overall reduction in dendritic length of NAc shell MSNs caused by long-term sucrose intake, results primarily from reductions in the complexity of distal branch orders. We observed reduced distal branching (4th and 5th order and above branch orders) and significantly reduced mean length at 5 th order and above dendrites, combined with increased spine densities at these branch orders. A common factor likely to influence this type of dendritic restructuring includes changes in synaptic connectivity and/or function (Russo et al., 2010). Previous studies have shown that glutamatergic synapses on MSNs are formed primarily on spines, particularly at distal dendrites (Groenewegen et al., 1999). Additionally, co-localization of dopamine and glutamatergic inputs from the prefrontal cortex (Sesack and Pickel, 1992), hippocampus (Totterdell and Smith, 1989; Sesack and Pickel, 1990), and amygdala (Johnson et al., 1994) have been observed on dendritic spines of MSNs. These observations combined with the increased spine density following long-term sucrose consumption seen in our study, support the formation of increased excitatory inputs. Therefore, the possibility arises where persistent effects caused by prolonged binge-like sucrose intake could facilitate increased excitatory synaptic activity at the distal dendrites of MSNs in the NAc shell. Consequently, reduction and/or retraction of distal dendrites may result via a synaptic homeostatic mechanism (Reissner and Kalivas, 2010), however this remains to be determined.

It is interesting to note that Crombag and colleagues showed that there was no spine density increase in the NAc shell following 4-week sucrose consumption via the nose-poke selfadministration paradigm despite a more robust acquisition and a higher response rate to sucrose when compared with amphetamine (Crombag et al., 2005). Their observation of an absence of change in spine density at 4 weeks mirrors our findings. By contrast, however, our study demonstrates that following long-term (12 week) exposure to chronic sucrose 
TABLE 3 | Branch order characteristics of medium spiny neurons from long-term sucrose and water drinking rats.

\begin{tabular}{|c|c|c|c|}
\hline Branch order properties & Water (9) & Sucrose (9) & Adjusted $P$-value \\
\hline 1st order branch segments & $4.6 \pm 0.1$ & $4.5 \pm 0.2$ & $P>0.9999$ \\
\hline 1st order mean branch segment length $(\mu \mathrm{m})$ & $25.9 \pm 3.5$ & $31.4 \pm 2.8$ & $P>0.9999$ \\
\hline 1st order branch spine density & $52.2 \pm 7.6$ & $67.8 \pm 6.6$ & $P=0.2129$ \\
\hline 2nd order branch segments & $8.5 \pm 0.3$ & $7.7 \pm 0.3$ & $P>0.9999$ \\
\hline 2nd order mean branch segment length ( $\mu \mathrm{m})$ & $65.2 \pm 6.0$ & $68.0 \pm 6.8$ & $P>0.9999$ \\
\hline 2nd order branch spine density & $41.3 \pm 3.6$ & $45.5 \pm 3.2$ & $P>0.9999$ \\
\hline 3rd order branch segments & $8.7 \pm 0.5$ & $8.1 \pm 0.7$ & $P>0.9999$ \\
\hline 3rd order mean branch segment length $(\mu \mathrm{m})$ & $71.1 \pm 6.7$ & $70.2 \pm 4.2$ & $P>0.9999$ \\
\hline 3rd order branch spine density & $40.2 \pm 5.6$ & $43.3 \pm 3.6$ & $P>0.9999$ \\
\hline 4th order branch segments & $5.2 \pm 0.9$ & $3.3 \pm 0.8$ & $P=0.0675$ \\
\hline 4th order mean branch segment length $(\mu \mathrm{m})$ & $56.9 \pm 6.6$ & $41.6 \pm 5.1$ & $P=0.3308$ \\
\hline 4th order and above branch spine density & $33.4 \pm 4.2$ & $52.5 \pm 6.8$ & $P=0.0271^{\star}$ \\
\hline 5th order and above branch segments & $3.3 \pm 0.7$ & $1.2 \pm 0.3$ & $P=0.0566$ \\
\hline 5th order and above mean branch segment length $(\mu \mathrm{m})$ & $53.9 \pm 7.2$ & $24.1 \pm 7.5$ & $P=0.0038^{\star *}$ \\
\hline
\end{tabular}

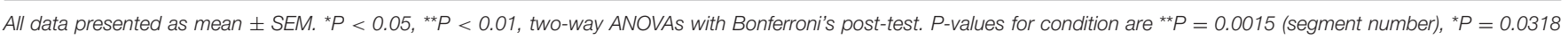
(mean segment length) and ${ }^{*} P=0.0124$ (spine density).

TABLE 4 | General morphologic parameters of medium spiny neurons from the nucleus accumbens core of short-term sucrose consuming rats and age matched water controls.

\begin{tabular}{lccc}
\hline Parameter & Water $(\boldsymbol{n})$ & Sucrose $(\boldsymbol{n})$ & $\boldsymbol{P}$-value \\
\hline Soma Volume $\left(\mu \mathrm{m}^{3}\right)$ & $2774 \pm 293(6)$ & $2885 \pm 92(6)$ & 0.7491 \\
Total Dendrite Length $(\mu \mathrm{m})$ & $1711 \pm 188(6)$ & $1793 \pm 132(6)$ & 0.7274 \\
Mean Tree Length $(\mu \mathrm{m})$ & $416 \pm 25(6)$ & $444 \pm 37(6)$ & 0.5688 \\
Nodes & $11.1 \pm 1.0(6)$ & $11.9 \pm 0.8(6)$ & 0.5928 \\
Endings & $15.3 \pm 1.4(6)$ & $16.2 \pm 0.8(6)$ & 0.6014 \\
Spines Per $100 \mu \mathrm{m}$ & $53.6 \pm 3.7(6)$ & $54.4 \pm 5.7(6)$ & 0.9142
\end{tabular}

All data presented as mean \pm SEM. Two-tailed unpaired Student's t-tests.

TABLE 5 | General morphologic parameters of medium spiny neurons from the nucleus accumbens core of long-term sucrose consuming rats and age matched water controls.

\begin{tabular}{lccc}
\hline Parameter & Water $(\boldsymbol{n})$ & Sucrose $(\boldsymbol{n})$ & $\boldsymbol{P}$-value \\
\hline Soma Volume $\left(\mu \mathrm{m}^{3}\right)$ & $4569 \pm 230(9)$ & $4862 \pm 372(9)$ & 0.5031 \\
Total Dendrite Length $(\mu \mathrm{m})$ & $1797 \pm 98(9)$ & $1775 \pm 120(9)$ & 0.8922 \\
Mean Tree Length $(\mu \mathrm{m})$ & $404 \pm 32(9)$ & $398 \pm 40(9)$ & 0.8980 \\
Nodes & $13.2 \pm 1.2(9)$ & $11.4 \pm 0.7(9)$ & 0.2091 \\
Endings & $18.2 \pm 1.4(9)$ & $16.2 \pm 0.9(9)$ & 0.2456 \\
Spines Per $100 \mu \mathrm{m}$ & $46.9 \pm 3.5(9)$ & $43.1 \pm 2.8(9)$ & 0.4130 \\
\hline
\end{tabular}

All data presented as mean \pm SEM. Two-tailed unpaired Student's t-tests.

consumption, there is a significant increase in distal spine density on the MSNs of the sucrose-experiences rats. Furthermore, our laboratory has previously shown that long-term (12 week) sucrose consumption facilitates a differential pharmacological response to pharmacotherapeutics that have been shown to modulate dopamine and acetylcholine responses at the level of the NAc (Shariff et al., in press). Taken together, this suggests that long-term (12 weeks and beyond) sucrose exposure, which is more accurately reflective of real-world scenarios, results in morphology adaptations at the level of the NAc.

In terms of drugs of abuse, repeated exposure to various drugs produces long-lasting changes in the structure of dendrites and dendritic spines. For example, amphetamines and cocaine both increase spine density in the NAc in both shell and core (Robinson and Kolb, 2004). Nicotine exposure has also been shown to increase spine density in the NAc shell. Conversely, morphine exposure leads to a decrease in spine density and dendritic branch complexity (Robinson and Kolb, 2004). In terms of long-term sucrose consumption, we observed an increase in spine density similar to amphetamine, cocaine and nicotine and opposite to the effect of morphine. However, unlike amphetamine and cocaine, but similar to nicotine, the increase of spine density on long-term exposure to sucrose is limited to the NAc shell. It is also interesting that changes in both dendritic branching (Robinson and Kolb, 1999) and spine density (Li et al., 2003) produced by amphetamine or cocaine are confined to distal dendrites of MSNs in the NAc, which reflects the findings in our study. Furthermore, and corroborative to the changes described above, sucrose consumption has also previously been shown to enhance excitatory synaptic strength onto accumbal dopamine neurons (Stuber et al., 2008b) as well as other components of the mesolimbic reward pathway (Stuber et al., 2008a; Chen et al., 2010). Taken together, this posits sucrose as a potent modulator of neuron morphology following prolonged heavy use, which is akin to the effects observed from drugs of abuse.

Although further investigations are required to uncover the cellular and synaptic mechanisms contributing to the morphological changes seen in this study, our results demonstrate significant neuronal effects engendered by longterm sucrose consumption. In particular, a consideration not examined in our study is whether the observed morphological effects of sucrose can also be elicited with non-caloric sweeteners such as saccharin. In this regard it is important to note that 
Lenoir and colleagues have shown that intense sweetness surpasses cocaine reward, be it generated by saccharin or sucrose (Lenoir et al., 2007). Furthermore, a recent study published by our lab (Shariff et al., in press) demonstrates that varenicline, a nicotinic acetylcholine receptor partial agonist reduced both sucrose and saccharin intake in rodents following the same long-term intermittent access regimen used in the present study. Interestingly, previous studies have shown similarities in the acute effects of non-caloric sweeteners such as saccharin and sucrose at the level of the NAc (Scheggi et al., 2013; Tukey et al., 2013; Carelli and West, 2014). However, further studies are need to determine whether non-caloric sweeteners can induce long-term effects similar to changes in the morphology of the NAc shell MSNs caused by long-term sucrose consumption reported here.

The lack of effect on NAc MSN morphology following short-term sucrose consumption, highlights the importance of implementing long-term studies to assess the impact of prolonged abuse of drugs or natural rewards like sucrose. In terms of dependence, not only are repeated cycles of binge intake and abstinence key components of the addiction cycle, a growing body of evidence has revealed that the transition to dependence is a progressive process that often occurs over an extended period of time. Although the addictive properties of sugars remain uncertain, the plausibility of addiction to other non-drug rewards such as sex, gambling and food is being increasingly investigated. The results from this study add merit to the hypothesis that sugars such as sucrose potentially have addictive properties following long-term, binge-like consumption. Our results also have implications for the growing number of children

\section{REFERENCES}

Ahmed, S. H., and Koob, G. F. (1998). Transition from moderate to excessive drug intake: change in hedonic set point. Science 282, 298-300. doi: 10.1126/science.282.5387.298

Ahmed, S. H., Lin, D., Koob, G. F., and Parsons, L. H. (2003). Escalation of cocaine self-administration does not depend on altered cocaine-induced nucleus accumbens dopamine levels. J. Neurochem. 86, 102-113. doi: 10.1046/j.14714159.2003.01833.x

Ahmed, S. H., Walker, J. R., and Koob, G. F. (2000). Persistent increase in the motivation to take heroin in rats with a history of drug escalation. Neuropsychopharmacology 22, 413-421. doi: 10.1016/S0893-133X(99)00 133-5

Arslanian, S. (2002). Type 2 diabetes in children: clinical aspects and risk factors. Horm Res 57 (Suppl. 1), 19-28. doi: 10.1159/000053308

Avena, N. M., Bocarsly, M. E., Hoebel, B. G., and Gold, M. S. (2011). Overlaps in the nosology of substance abuse and overeating: the translational implications of "food addiction". Curr. Drug Abuse Rev. 4, 133-139. doi: $10.2174 / 1874473711104030133$

Avena, N. M., Rada, P., and Hoebel, B. G. (2008). Evidence for sugar addiction: behavioral and neurochemical effects of intermittent, excessive sugar intake. Neurosci. Biobehav. Rev. 32, 20-39. doi: 10.1016/j.neubiorev.2007.04.019

Avena, N. M., Rada, P., Moise, N., and Hoebel, B. G. (2006). Sucrose sham feeding on a binge schedule releases accumbens dopamine repeatedly and eliminates the acetylcholine satiety response. Neuroscience $139,813-820$. doi: 10.1016/j.neuroscience.2005.12.037

Benton, D. (2010). The plausibility of sugar addiction and its role in obesity and eating disorders. Clin. Nutr. 29, 288-303. doi: 10.1016/j.clnu.2009.12.001 and adolescents who maintain unhealthy eating habits (high sugar consumption and binge eating) into adulthood. In line with the increased risk of developing metabolic effects it is also possible that neurological and psychiatric consequences affecting mood and motivation may also result from these behaviors.

\section{AUTHOR CONTRIBUTIONS}

Participated in research design: $\mathrm{PK}, \mathrm{SB}$. Conducted experiments: PK, MS, AB, MF, EM. Data analysis: PK, MF, MS. Interpreted the data and contributed to writing of the manuscript: PK, MS, MF, $\mathrm{EM}, \mathrm{MB}, \mathrm{SB}$. All authors read and approved the final manuscript for submission.

\section{ACKNOWLEDGMENTS}

This work was supported by funding from grants from the Australian Research Council (FT1110884) to SB and the National Health and Medical Research Council (1061979) to SB and MB.

\section{SUPPLEMENTARY MATERIAL}

The Supplementary Material for this article can be found online at: http://journal.frontiersin.org/article/10.3389/fnbeh. 2016.00054

Supplementary Figure 1 | Sucrose intake and preference from 4 and 12 week sucrose consuming rats. (A,B) show escalation in total sucrose intake $(\mathrm{ml})$ over 4 and 12 weeks of exposure. (C,D) show high preference for sucrose over water during periods of sucrose presentation.

Bray, G. A., and Popkin, B. M. (2014). Dietary sugar and body weight: have we reached a crisis in the epidemic of obesity and diabetes?: health be damned! Pour on the sugar. Diabetes Care 37, 950-956. doi: 10.2337/dc13-2085

Brog, J. S., Salyapongse, A., Deutch, A. Y., and Zahm, D. S. (1993). The patterns of afferent innervation of the core and shell in the "accumbens" part of the rat ventral striatum: immunohistochemical detection of retrogradely transported fluoro-gold. J. Comp. Neurol. 338, 255-278. doi: 10.1002/cne.903380209

Bucher Della Torre, S., Keller, A., Laure Depeyre, J., and Kruseman, M. (2015). Sugar-sweetened beverages and obesity risk in children and adolescents: a systematic analysis on how methodological quality may influence conclusions. J. Acad. Nutr. Diet. doi: 10.1016/j.jand.2015.05.020. [Epub ahead of print].

Carelli, R. M., and West, E. A. (2014). When a good taste turns bad: neural mechanisms underlying the emergence of negative affect and associated natural reward devaluation by cocaine. Neuropharmacology 76 (Pt B), 360-369. doi: 10.1016/j.neuropharm.2013.04.025

Chen, B. T., Hopf, F. W., and Bonci, A. (2010). Synaptic plasticity in the mesolimbic system: therapeutic implications for substance abuse. Ann. N.Y. Acad. Sci. 1187, 129-139. doi: 10.1111/j.1749-6632.2009.05154.x

Colantuoni, C., Rada, P., McCarthy, J., Patten, C., Avena, N. M., Chadeayne, A., et al. (2002). Evidence that intermittent, excessive sugar intake causes endogenous opioid dependence. Obes. Res. 10, 478-488. doi: 10.1038 /oby. 2002.66

Colantuoni, C., Schwenker, J., McCarthy, J., Rada, P., Ladenheim, B., Cadet, J. L., et al. (2001). Excessive sugar intake alters binding to dopamine and mu-opioid receptors in the brain. Neuroreport 12, 3549-3552. doi: 10.1097/00001756200111160-00035

Crombag, H. S., Gorny, G., Li, Y., Kolb, B., and Robinson, T. E. (2005). Opposite effects of amphetamine self-administration experience on dendritic spines 
in the medial and orbital prefrontal cortex. Cereb. Cortex 15, 341-348. doi: $10.1093 /$ cercor/bhh136

Dabelea, D., Mayer-Davis, E. J., Saydah, S., Imperatore, G., Linder, B., Divers, J., et al. (2014). Prevalence of type 1 and type 2 diabetes among children and adolescents from 2001 to 2009. JAMA 311, 1778-1786. doi: 10.1001/jama.2014.3201

Davis, C. (2015). The epidemiology and genetics of binge eating disorder (BED). CNS Spectr. 20, 522-529. doi: 10.1017/s1092852915000462

Ford, E. S., and Dietz, W. H. (2013). Trends in energy intake among adults in the United States: findings from NHANES. Am. J. Clin. Nutr. 97, 848-853. doi: 10.3945/ajcn.112.052662

Fryar, C. D., Carroll, M. D., and Ogden, C. L. (2014). Prevalence of Overweight, Obesity, and Extreme Obesity Among Adults: United States, 1960-1962 through 2011-2012. Atlanta, GA: CDC.

Graveland, G. A., and DiFiglia, M. (1985). The frequency and distribution of medium-sized neurons with indented nuclei in the primate and rodent neostriatum. Brain Res. 327, 307-311. doi: 10.1016/0006-8993(85)91524-0

Groenewegen, H. J., Wright, C. I., Beijer, A. V., and Voorn, P. (1999). Convergence and segregation of ventral striatal inputs and outputs. Ann. N.Y. Acad. Sci. 877, 49-63. doi: 10.1111/j.1749-6632.1999.tb09260.x

Groves, P. M. (1980). Synaptic endings and their postsynaptic targets in neostriatum: synaptic specializations revealed from analysis of serial sections. Proc. Natl. Acad. Sci. U.S.A. 77, 6926-6929. doi: 10.1073/pnas.77.11.6926

Groves, P. M., Linder, J. C., and Young, S. J. (1994). 5-hydroxydopamine-labeled dopaminergic axons: three-dimensional reconstructions of axons, synapses and postsynaptic targets in rat neostriatum. Neuroscience 58, 593-604. doi: 10.1016/0306-4522(94)90084-1

$\mathrm{Hu}, \mathrm{F}$. B. (2013). Resolved: there is sufficient scientific evidence that decreasing sugar-sweetened beverage consumption will reduce the prevalence of obesity and obesity-related diseases. Obes. Rev. 14, 606-619. doi: 10.1111/obr. 12040

Johnson, L. R., Aylward, R. L., Hussain, Z., and Totterdell, S. (1994). Input from the amygdala to the rat nucleus accumbens: its relationship with tyrosine hydroxylase immunoreactivity and identified neurons. Neuroscience 61, 851-865. doi: 10.1016/0306-4522(94)90408-1

Kaiya, H., and Namba, M. (1981). Two types of dopaminergic nerve terminals in the rat neostriatum. An ultrastructural study. Neurosci. Lett. 25, 251-256. doi: 10.1016/0304-3940(81)90400-6

Kalivas, P. W., and Miller, J. S. (1984). Neurotensin neurons in the ventral tegmental area project to the medial nucleus accumbens. Brain Res. 300, 157-160. doi: 10.1016/0006-8993(84)91351-9

Kawaguchi, Y., Wilson, C. J., and Emson, P. C. (1990). Projection subtypes of rat neostriatal matrix cells revealed by intracellular injection of biocytin. J. Neurosci. 10, 3421-3438.

Kelley, A. E., and Swanson, C. J. (1997). Feeding induced by blockade of AMPA and kainate receptors within the ventral striatum: a microinfusion mapping study. Behav. Brain Res. 89, 107-113. doi: 10.1016/S0166-4328(97)00054-5

Kemp, J. M., and Powell, T. P. (1971). The synaptic organization of the caudate nucleus. Philos. Trans. R. Soc. Lond. B Biol. Sci. 262, 403-412. doi: $10.1098 /$ rstb. 1971.0103

Kenny, P. J. (2011). Reward mechanisms in obesity: new insights and future directions. Neuron 69, 664-679. doi: 10.1016/j.neuron.2011.02.016

Kessler, R. C., Berglund, P. A., Chiu, W. T., Deitz, A. C., Hudson, J. I., Shahly, V., et al. (2013). The prevalence and correlates of binge eating disorder in the World Health Organization World Mental Health Surveys. Biol. Psychiatry 73, 904-914. doi: 10.1016/j.biopsych.2012.11.020

Klenowski, P. M., Fogarty, M. J., Belmer, A., Noakes, P. G., Bellingham, M. C., and Bartlett, S. E. (2015). Structural and functional characterization of dendritic arbors and GABAergic synaptic inputs on interneurons and principal cells in the rat basolateral amygdala. J. Neurophysiol. 114, 942-957. doi: 10.1152/jn.00824.2014

Lenoir, M., Serre, F., Cantin, L., and Ahmed, S. H. (2007). Intense sweetness surpasses cocaine reward. PLOS ONE 2:e698. doi: 10.1371/journal.pone.0000698

Li, Y., Kolb, B., and Robinson, T. E. (2003). The location of persistent amphetamine-induced changes in the density of dendritic spines on medium spiny neurons in the nucleus accumbens and caudate-putamen. Neuropsychopharmacology 28, 1082-1085. doi: 10.1038/sj.npp.1300115
Lindvall, O., and Björklund, A. (1978). Anatomy of the dopaminergic neuron systems in the rat brain. Adv. Biochem. Psychopharmacol. 19, 1-23.

Lutter, M., and Nestler, E. J. (2009). Homeostatic and hedonic signals interact in the regulation of food intake. J. Nutr. 139, 629-632. doi: 10.3945/jn.108.0 97618

Maldonado-Irizarry, C. S., Swanson, C. J., and Kelley, A. E. (1995). Glutamate receptors in the nucleus accumbens shell control feeding behavior via the lateral hypothalamus. J. Neurosci. 15, 6779-6788.

Malik, V. S., Popkin, B. M., Bray, G. A., Després, J. P., and Hu, F. B. (2010). Sugar-sweetened beverages, obesity, type 2 diabetes mellitus, and cardiovascular disease risk. Circulation 121, 1356-1364. doi: 10.1161/CIRCULATIONAHA.109.876185

Ng, S. W., Slining, M. M., and Popkin, B. M. (2012). Use of caloric and noncaloric sweeteners in US consumer packaged foods, 2005-2009. J. Acad. Nutr. Diet. 112, 1828-1834 e1821-e1826. doi: 10.1016/j.jand.2012.07.009

Nielsen, S. J., Siega-Riz, A. M., and Popkin, B. M. (2002). Trends in energy intake in U.S. between 1977 and 1996: similar shifts seen across age groups. Obes. Res. 10, 370-378. doi: 10.1038/oby.2002.51

Paxinos, G., and Watson, C. (2007). The Rat Brain in Stereotaxic Coordinates. Amsterdam; Boston, MA: Academic Press/Elsevier.

Popkin, B. M. (2010). What's Wrong with the U.S. Approach to Obesity? Virtual Mentor 12, 316-320. doi: 10.1001/virtualmentor.2010.12.4.pfor2-1004

Rada, P., Avena, N. M., and Hoebel, B. G. (2005). Daily bingeing on sugar repeatedly releases dopamine in the accumbens shell. Neuroscience 134, 737-744. doi: 10.1016/j.neuroscience.2005.04.043

Rafols, J. A., Cheng, H. W., and McNeill, T. H. (1989). Golgi study of the mouse striatum: age-related dendritic changes in different neuronal populations. $J$. Comp. Neurol. 279, 212-227. doi: 10.1002/cne.902790205

Ranjan, A., and Mallick, B. N. (2010). A modified method for consistent and reliable Golgi-cox staining in significantly reduced time. Front. Neurol. 1:157. doi: $10.3389 /$ fneur.2010.00157

Reinehr, T. (2013). Type 2 diabetes mellitus in children and adolescents. World J. Diabetes 4, 270-281. doi: 10.4239/wjd.v4.i6.270

Reissner, K. J., and Kalivas, P. W. (2010). Using glutamate homeostasis as a target for treating addictive disorders. Behav. Pharmacol. 21, 514-522. doi: 10.1097/FBP.0b013e32833d41b2

Reynolds, S. M., and Berridge, K. C. (2003). Glutamate motivational ensembles in nucleus accumbens: rostrocaudal shell gradients of fear and feeding. Eur. J. Neurosci. 17, 2187-2200. doi: 10.1046/j.1460-9568.2003.02642.x

Richard, J. M., and Berridge, K. C. (2011). Nucleus accumbens dopamine/glutamate interaction switches modes to generate desire versus dread: $\mathrm{D}(1)$ alone for appetitive eating but $\mathrm{D}(1)$ and $\mathrm{D}(2)$ together for fear. J. Neurosci. 31, 12866-12879. doi: 10.1523/JNEUROSCI.1339-11.2011

Robinson, T. E., and Kolb, B. (1999). Alterations in the morphology of dendrites and dendritic spines in the nucleus accumbens and prefrontal cortex following repeated treatment with amphetamine or cocaine. Eur. J. Neurosci. 11, 1598-1604. doi: 10.1046/j.1460-9568.1999.00576.x

Robinson, T. E., and Kolb, B. (2004). Structural plasticity associated with exposure to drugs of abuse. Neuropharmacology 47(Suppl. 1), 33-46. doi: 10.1016/j.neuropharm.2004.06.025

Russo, S. J., Dietz, D. M., Dumitriu, D., Morrison, J. H., Malenka, R. C., and Nestler, E. J. (2010). The addicted synapse: mechanisms of synaptic and structural plasticity in nucleus accumbens. Trends Neurosci. 33, 267-276. doi: 10.1016/j.tins.2010.02.002

Rutledge, L. T., Duncan, J., and Beatty, N. (1969). A study of pyramidal cell axon collaterals in intact and partially isolated adult cerebral cortex. Brain Res. 16, 15-22. doi: 10.1016/0006-8993(69)90082-1

Saper, C. B., Chou, T. C., and Elmquist, J. K. (2002). The need to feed: homeostatic and hedonic control of eating. Neuron 36, 199-211. doi: 10.1016/S08966273(02)00969-8

Scheggi, S., Secci, M. E., Marchese, G., De Montis, M. G., and Gambarana, C. (2013). Influence of palatability on motivation to operate for caloric and noncaloric food in non food-deprived and food-deprived rats. Neuroscience 236, 320-331. doi: 10.1016/j.neuroscience.2013.01.027

Sesack, S. R., and Pickel, V. M. (1990). In the rat medial nucleus accumbens, hippocampal and catecholaminergic terminals converge on spiny neurons and are in apposition to each other. Brain Res. 527, 266-279. doi: 10.1016/00068993(90)91146-8 
Sesack, S. R., and Pickel, V. M. (1992). Prefrontal cortical efferents in the rat synapse on unlabeled neuronal targets of catecholamine terminals in the nucleus accumbens septi and on dopamine neurons in the ventral tegmental area. J. Comp. Neurol. 320, 145-160. doi: 10.1002/cne.903200202

Shariff, M., Quik, M., Holgate, J. Y., Morgan, M., Patkar, O. L., Tam, V., et al. (in press). Neuronal nicotinic acetylcholine receptor modulators reduce sugar intake. PLOS ONE.

Sheehan, D. V., and Herman, B. K. (2015). The psychological and medical factors associated with untreated binge eating disorder. Prim. Care Companion CNS Disord. 17. doi: 10.4088/PCC.14r01732

Simms, J. A., Steensland, P., Medina, B., Abernathy, K. E., Chandler, L. J., Wise, R., et al. (2008). Intermittent access to $20 \%$ ethanol induces high ethanol consumption in Long-Evans and Wistar rats. Alcohol. Clin. Exp. Res. 32, 1816-1823. doi: 10.1111/j.1530-0277.2008.00753.x

Steensland, P., Simms, J. A., Holgate, J., Richards, J. K., and Bartlett, S. E. (2007). Varenicline, an alpha4beta2 nicotinic acetylcholine receptor partial agonist, selectively decreases ethanol consumption and seeking. Proc. Natl. Acad. Sci. U.S.A. 104, 12518-12523. doi: 10.1073/pnas.0705368104

Stuber, G. D., Hopf, F. W., Hahn, J., Cho, S. L., Guillory, A., and Bonci, A. (2008a). Voluntary ethanol intake enhances excitatory synaptic strength in the ventral tegmental area. Alcohol. Clin. Exp. Res. 32, 1714-1720. doi: 10.1111/j.15300277.2008.00749.x

Stuber, G. D., Klanker, M., de Ridder, B., Bowers, M. S., Joosten, R. N., Feenstra, M. G., et al. (2008b). Reward-predictive cues enhance excitatory synaptic strength onto midbrain dopamine neurons. Science 321, 1690-1692. doi: 10.1126/science. 1160873

Swanson, S. A., Crow, S. J., Le Grange, D., Swendsen, J., and Merikangas, K. R. (2011). Prevalence and correlates of eating disorders in adolescents. Results from the national comorbidity survey replication adolescent supplement. Arch. Gen. Psychiatry 68, 714-723. doi: 10.1001/archgenpsychiatry.2011.22

Te Morenga, L., Mallard, S., and Mann, J. (2013). Dietary sugars and body weight: systematic review and meta-analyses of randomised controlled trials and cohort studies. BMJ 346:e7492. doi: 10.1136/bmj.e7492

Totterdell, S., and Smith, A. D. (1989). Convergence of hippocampal and dopaminergic input onto identified neurons in the nucleus accumbens of the rat. J. Chem. Neuroanat. 2, 285-298.
Tukey, D. S., Ferreira, J. M., Antoine, S. O., D’Amour, J. A., Ninan, I., Cabeza de Vaca, S., et al. (2013). Sucrose ingestion induces rapid AMPA receptor trafficking. J. Neurosci. 33, 6123-6132. doi: 10.1523/JNEUROSCI.4806-12.2013

Veening, J. G., Cornelissen, F. M., and Lieven, P. A. (1980). The topical organization of the afferents to the caudatoputamen of the rat. A horseradish peroxidase study. Neuroscience 5, 1253-1268. doi: 10.1016/03064522(80)90198-0

Ventura, T., Santander, J., Torres, R., and Contreras, A. M. (2014). Neurobiologic basis of craving for carbohydrates. Nutrition 30, 252-256. doi: 10.1016/j.nut.2013.06.010

Volkow, N. D., Wang, G. J., Fowler, J. S., Tomasi, D., and Baler, R. (2012). Food and drug reward: overlapping circuits in human obesity and addiction. Curr. Top. Behav. Neurosci. 11, 1-24. doi: 10.1007/7854_2011_169

Wise, R. A. (1973). Voluntary ethanol intake in rats following exposure to ethanol on various schedules. Psychopharmacologia 29, 203-210. doi: 10.1007/BF00414034

Wright, C. I., and Groenewegen, H. J. (1995). Patterns of convergence and segregation in the medial nucleus accumbens of the rat: relationships of prefrontal cortical, midline thalamic, and basal amygdaloid afferents. J. Comp. Neurol. 361, 383-403. doi: 10.1002/cne.903610304

Conflict of Interest Statement: The authors declare that the research was conducted in the absence of any commercial or financial relationships that could be construed as a potential conflict of interest.

The reviewers SC, SA and handling Editor declared their shared affiliation, and the handling Editor states that the process nevertheless met the standards of a fair and objective review.

Copyright (c) 2016 Klenowski, Shariff, Belmer, Fogarty, Mu, Bellingham and Bartlett. This is an open-access article distributed under the terms of the Creative Commons Attribution License (CC BY). The use, distribution or reproduction in other forums is permitted, provided the original author(s) or licensor are credited and that the original publication in this journal is cited, in accordance with accepted academic practice. No use, distribution or reproduction is permitted which does not comply with these terms. 